\title{
Pravastatin and cancer: an unproven association
}

\author{
James M. Brophy
}

$\infty$

See related article page 649

$\mathrm{S}$ tatins produce large cardiovascular benefits, particularly in the secondary prevention of cardiovascular disease. In a meta-analysis of I4 randomized trials, participants with a history of heart disease who were taking statins for 5 years had 48 fewer vascular events per 1000 than those in the control group. ${ }^{1}$ Importantly, cardiovascular events occurred about 3 times more frequently than cases of cancer in these trials. In this issue, Bonovas and Sitaras report a possible association between the use of pravastatin (a hydrophilic statin) and cancer among elderly patients in their meta-analysis of I2 randomized controlled trials. ${ }^{2}$ Their study is generally well performed and follows the QUOROM guidelines for meta-analyses; however, readers may have several questions about the clinical significance of these findings. Although one may choose to simply dismiss subgroup analyses as merely being hypothesis generating, a Bayesian paradigm, which explicitly states prior beliefs and incorporates new findings according to the rules of probability, may provide additional insights for interpreting this new evidence.

\section{What are our prior beliefs?}

Previous meta-analyses of randomized controlled trials that included all types of statins have not demonstrated any increased risk of cancer. ${ }^{1,3}$ The present study also does not demonstrate an increase in the overall cancer risk associated with pravastatin therapy. This study was performed before the results were available from the Management of Elevated Cholesterol in the Primary Prevention Group of Adult Japanese (MEGA) trial, ${ }^{4}$ which followed 8000 Japanese people (mean age 58 years) randomly assigned to receive either pravastatin and diet or diet alone for more than 5 years. Again there was no increase in cancer risk associated with pravastatin therapy (risk ratio $0.97,95 \%$ confidence interval $0.76-\mathrm{I} .25, p=0.8 \mathrm{I}$ ).

Several observational studies have suggested that statins are associated not with an increase, but rather with a decrease in the risk of cancer. ${ }^{5,6}$ Recently, 2 large observational studies that examined over 7500 incident breast cancer cases found no increased risk of cancer with statin therapy and perhaps even a small benefit of lipophilic agents. ${ }^{7,8}$ Although these studies grouped multiple statins together and were not randomized, the number of observed breast cancer cases was 2.5 times greater than the total number of unspecified cancer cases reported in the randomized controlled trials. Concerns of bias and residual confounding are always issues in observational research. However, these concerns are more important in studies of intended as opposed to unintended effects. For example, several high-quality observational studies of the cardiovascular risk associated with cyclooxygenase-2 (COX-2) inhibitors have recently confirmed the increased risks observed in randomized controlled trials. ${ }^{9}$

The incidence rates for individual types of cancer among patients taking pravastatin therapy have not been reported, and the biological plausibility that pravastatin therapy can initiate or promote all types of cancer seems unlikely. Therefore, although the impact of age on the incidence of all cancers among statin users has not been directly studied previously, my prior belief (probability) in the carcinogenicity of pravastatin is relatively low, perhaps less than $1 \%$ to $5 \%$, equivalently expressed as I:99 or I:I9 odds respectively.

\section{The present study}

In the study by Bonovas and Sitaras, the overall association between pravastatin use and cancer was not statistically significant. However, using meta-regression analysis, the authors have dissected out a potential risk in elderly patients. This analysis involves a linear regression model developed from the individual trials, with log relative risk as the dependent variable and mean age as the independent variable. The authors report the slope coefficient of the log relative risk on mean age to be $0.014(p=0.006)$; this translates to a risk ratio of $\mathrm{I} .22$ for a trial with a mean participant age of 75 years.

How confident are we of this regression equation? Over 42000 patients were included in the original randomized controlled trials, yet the sample size for the meta-regression was reduced to the 13 available trials. As an analogy, consider a regression equation between the risks of cancer as a function of age based on 13 people, even if their risk was measured repeatedly. Furthermore, imagine that the age of these I3 people was not measured and instead only the average age of people living in their neighbourhood was used. Differences in other factors (e.g., sex, body mass index, smoking status) may have contributed to overall trial heterogeneity, yet these factors have not been, and indeed cannot be, assessed in this small sample size. In other words, a multivariable analysis to discover independent effects is impossible.

Unfortunately, the small $p$ value calculated for the slope coefficient does not provide sufficient reassurance for the above concerns and in no way addresses model uncertainty including the relative weights assigned to each trial. The authors claim that their results are robust to the removal of the PROSPER trial; however, the relation between age and cancer risk beyond the age of 69 would depend entirely on extrapolation, which has inherent hazards. This is not to imply that the authors' model is incorrect, but merely to demonstrate that the reported results are perhaps not as robust as suggested by the small $p$ value. 
Bonovas and Sitaras expressed appropriate concern and tested for publication bias, which is a serious potential problem in meta-analyses. However, they included only randomized controlled trials that reported cancer rates and as a result excluded at least 2 other randomized controlled trials of pravastatin therapy that did not report cancer rates. ${ }^{10,11}$ Since other clinical events were reported in these 2 studies, one may assume that the absence of reported cancer rates equates to an absence of differences. The inclusion of these 2 additional studies may well have influenced the final conclusions, and an effort to obtain these missing data would have been helpful.

The potential bias of attributing group characteristics to individuals (ecology fallacy), combined with the exclusion of trials, the small study size and model uncertainty, should surely influence our degree of confidence in a relation between cancer risk due to pravastatin therapy and age. Given these limitations, I would estimate the likelihood ratio (the probability of obtaining the results given a true association divided by the probability of obtaining the results if no association exists) to be no different than I. Although some may believe this to be a harsh judgment of the present study, a recent report has suggested that most published research findings are actually false. ${ }^{12}$

\section{What should we now believe?}

Bonovas and Sitaras are rightly reserved in their conclusions, are careful not to overstate their results and call judiciously for further research. Overemphasizing the age-related results while ignoring or minimizing the overall results would be analogous to the inappropriate emphasis occasionally attached to subgroup analysis. As readers, we should be similarly circumspect in our interpretation.

To return to Bayes' theorem, remember that the posterior odds (probability after the study results) equals the prior odds (probability before the study results) multiplied by the likelihood ratio. Therefore, given that my prior odds for this association were low, my posterior odds remain equally low, in the range of only $\mathrm{I} \%-5 \%$ probability, that the reported association is in fact true. Although this may be enough to stimulate further research into this question, it seems reasonable to conclude that a relation between pravastatin and cancer re- mains unproven and that the data at present are insufficient to change clinical practice.

\section{This article has been peer reviewed.}

James Brophy is an Associate Professor of Medicine, McGill University and Université de Montréal, and is Director of the Technology Assessment Unit for the McGill University Health Centre and the Centre Hospitalier du Université de Montréal, Montréal, Que.

Competing interests: None declared.

Acknowledgement: James Brophy receives financial support from le Fonds de la recherche en santé du Québec.

\section{REFERENCES}

I. Baigent C, Keech A, Kearney PM, et al; Cholesterol Treatment Trialists' (CTT) Collaborators. Efficacy and safety of cholesterol-lowering treatment: prospective meta-analysis of data from 90,056 participants in 14 randomised trials of statins [published erratum in Lancet 2005;366:1358]. Lancet 2005;366:1267-78.

2. Bonovas S, Sitaras NM. Does pravastatin promote cancer in elderly patients? A meta-analysis. $C M A J$ 2007;176(5):649-54.

3. Dale KM, Coleman CI, Henyan NN, et al. Statins and cancer risk: a meta-analysis. JAMA 2006;295:74-80.

4. Nakamura $\mathrm{H}$, Arakawa $\mathrm{K}$, Itakura $\mathrm{H}$, et al. Primary prevention of cardiovascular disease with pravastatin in Japan (MEGA Study): a prospective randomised controlled trial. Lancet 2006;368:1155-63.

5. Blais L, Desgagne A, LeLorier J. 3-Hydroxy-3-methylglutaryl coenzyme A reductase inhibitors and the risk of cancer: a nested case-control study. Arch Intern Med 2000; $160: 2363-8$.

6. Poynter JN, Gruber SB, Higgins PDR, et al. Statins and the risk of colorectal cancer. NEngl JMed 2005;352:2184-92.

7. Cauley JA, McTiernan A, Rodabough RJ, et al. Statin use and breast cancer: prospective results from the Women's Health Initiative. J Natl Cancer Inst 2006;08:700-7.

8. Eliassen AH, Colditz GA, Rosner B, et al. Serum lipids, lipid-lowering drugs, and the risk of breast cancer. Arch Intern Med 2005;165:2264-7I.

9. Brophy JM. Celecoxib and cardiovascular risks. Expert Opin Drug Saf 2005;4: I005-I5.

Io. Tamura A, Mikuriya Y, Nasu M; Coronary Artery Regression Study (CARS) Group. Effect of pravastatin (Io $\mathrm{mg} /$ day) on progression of coronary atherosclerosis in patients with serum total cholesterol levels from $\mathrm{r} 60$ to $220 \mathrm{mg} / \mathrm{dl}$ and angiographically documented coronary artery disease. Am J Cardiol 1997;79:893-6.

II. Pravastatin Multinational Study Group for Cardiac Risk Patients. Effects of pravastatin in patients with serum total cholesterol levels from 5.2 to $7.8 \mathrm{mmol} /$ liter (200 to $300 \mathrm{mg} / \mathrm{dl}$ ) plus two additional atherosclerotic risk factors. Am J Cardiol 1993 ; 72:103I-7.

I2. Ioannidis JPA. Why most published research findings are false. PLoS Med 2005;2:eI24

Correspondence to: Dr. James M. Brophy, Divisions of Cardiology and Clinical Epidemiology, Rm. R4.I2, McGill University Health Centre, Royal Victoria Hospital, 687 Pine Ave. W, Montréal QC H3A IAI; fax 519-843-I493; james.brophy@mcgill.ca

\section{LEADERSHIP}

$C M A J$ is a founding member of the International Committee of Medical Journal Editors, an organization that is devoted to ensuring the highest integrity in scientific publishing and is a driving force in the mandatory registration of clinical trials. 